\title{
TOTAL REPAIR COST LIMIT REPLACEMENT POLICIES
}

FRANK BEICHELT

\author{
University of the Witwatersrand \\ Department of Statistics and Actuarial Science \\ Johannesburg \\ South Africa
}

\begin{abstract}
Practical applications of stochastic maintenance models frequently fail due to insufficient information on the lifetime distribution of the systems. Moreover, the structure of numerous maintenance policies which have been proposed up till now is so complicated that they have purely academic significance. This paper presents a policy for optimal scheduling of replacement intervals where decisions are based only on the maintenance cost parameters. The simple structure of this policy and the circumstance that the cost parameters are usually known facilitates its practical application.
\end{abstract}

\section{INTRODUCTION}

A common maintenance policy for technical systems consists in replacing a system by a new one at that moment $\tau^{*}$, where the expected long-run maintenance cost rate (shortly: maintenance cost rate) is minimal CLAPHAM [4], SMITH [5]. $\tau^{*}$ is called the economic lifetime. But the strict application of the economic lifetime does not take into account individual deviations of repair cost rates of single systems from the average cost development. Thus the repair cost limit replacement policy has been suggested: When a failure occurs, the necessary repair cost is estimated. If this cost exceeds a certain amount, called the repair cost limit, the system is not repaired but replaced. A policy of this structure was proposed by GARDENT and NONANT [6] and was extended and successfully applied by DRINKWATER and HASTINGS [7] (for a survey 
modifications and a mathematical treatment see BEICHELT [1]). Examples have shown that applying a repair cost limit which is optimal with respect to the maintenance cost rate can be more efficient than applying the economic lifetime, see WHITE [8]. A disadvantage of the repair cost limit replacement policy is that the replacement decision depends only on the cost of a single repair. Long-lasting situations characterized by frequent repairs whose costs are all below the repair cost limit do not directly influence the moment of replacement, although the total repair cost rate might justify a replacement. Thus, further financial savings seem to be possible if the replacement decision depends on the whole history of the repair process. Following this idea, the paper proposes maintenance policies by bounding the repair cost rate and the total repair cost, respectively, within a replacement cycle.

\section{Assumptions}

1) The planning horizon is infinite.

2) Replacements and repairs take only negligible time.

3) The replacement intervals (time spans between successive replacements) are i.i.d. positive random variables with finite expectation.

\section{LIMIT FOR THE REPAIR COST RATE}

Let $A(t)$ be the total repair cost in the interval $(0, t]$. Then the repair cost rate is defined as $Z(t)=A(t) / t, t>0$

Policy I The system is replaced as soon as the actual repair cost rate $\mathrm{Z}(\mathrm{t})$ reaches or exceeds a critical level $z$.

If $A(t)$ denotes the total repair cost in the interval $(0, t]$, then $Z(t)=A(t) / t$.

According to policy $I$, the random replacement interval $Y_{Z}(z)$ is a first passage time of $\{Z(t), t \geq 0\}$ :

$$
Y_{Z}(z)=\inf _{t}\{t ; Z(t) \geq z\}
$$

Assuming a constant replacement cost $\mathrm{C}$, the maintenance cost rate is given by

$$
K(z)=z+\frac{C}{E\left(Y_{Z}(z)\right)}
$$

This is a straightforward corollary of the elementary renewal theorem. 
The repair cost processes $\{Z(t), t \geq 0\}$ considered in what follows will be functionals of the Wiener process with drift $\{\mathrm{W}(\mathrm{t}), \mathrm{t} \geq 0\}$. This process is defined by three characteristic properties:

1) $W(0)=0$.

2) $\{W(t), t \geq 0\}$ has homogeneous and independent increments.

3) For all $t>0, W(t)$ is normally distributed with $E(W(t))=\mu t$ and $\operatorname{Var}(W(t))=\sigma^{2} t$.

The constant $\mu$ is called the drift parameter. $\{\mathrm{W}(\mathrm{t}), \mathrm{t} \geq 0\}$ is said to have a positve drift if $\mu>0$. Otherwise it has a negative drift. If $\mu=0$, then $\{W(t), t \geq 0\}$ becomes the Wiener process or the process of Brownian motion.

Let $Y_{W}(w)$ denote the first passage time of the Wiener process with drift with respect to the constant level $w$. Then it is well-known that $Y_{W}(w)$ has an inverse Gaussian distribution with probability density (see, e.g. BEICHELT and FATTI [2], CHHIKARA and FOLKS [3])

$$
f_{Y_{W}(w)}(t)=\frac{w}{\sqrt{2 \pi} \sigma t^{3 / 2}} \exp \left\{-\frac{(w-\mu t)^{2}}{2 \sigma^{2} t}\right\},
$$

where

$$
t>0, \mu \neq 0, \operatorname{sign}(\mu)=\operatorname{sign}(w) \text {. }
$$

Hence the expected value of the first passage time of the Wiener process with drift with respect to the level $w$ is

$$
E\left(Y_{W}(w)\right)=\frac{|w|}{|\mu|}
$$

Example 1 Let $Z(t)$ be given by

$$
\mathrm{Z}(\mathrm{t})=\mathrm{z}_{0} \mathrm{e}^{\mathrm{W}(\mathrm{t})}, \quad \mathrm{z}_{0}>0,
$$

where $\{W(t), t \geq 0\}$ is a Wiener process with positive drift. Since $Z(t)=z$ holds if and only if

$$
W(t)=\ln \frac{z}{z_{0}}
$$

the expected first passage time of the process $\{Z(t), t \geq 0\}$ with respect to $z$ is according to (3)

$$
E\left(Y_{Z}(z)\right)=\frac{\ln \left(Z / Z_{0}\right)}{\mu}
$$


Hence, the corresponding maintenance cost rate is

$$
K(z)=z+\frac{\mu C}{\ln \left(z / z_{0}\right)} .
$$

The optimal $z=z^{*}$ satisfies the equation $d K(z) / d z=0$ :

$$
z\left(\ln z / z_{0}\right)^{2}=\mu C .
$$

The corresponding minimal maintenance cost rate becomes

$$
\mathrm{K}(\mathrm{Z} *)=\mathrm{Z} *+\sqrt{\mu \mathrm{CZ} *} \text {. }
$$

Comparision with the economic lifetime approach: If the system is always replaced after a constant time span $\tau$, the maintenance cost rate is

$$
K(\tau)=E(Z(\tau))+\frac{C}{\tau},
$$

where

$$
E(Z(t))=z_{0} e^{\beta t}
$$

with

$$
\beta=\mu+\sigma^{2} / 2 \text { and } \sigma^{2}=\operatorname{Var}(W(1)) \text {. }
$$

The economic lifetime $\tau=\tau^{*}$ is the unique solution of the equation

$$
\tau^{2} e^{b \tau}=\frac{C}{z_{0} \beta} .
$$

The corresponding minimum maintenance cost rate becomes

$$
K(\tau *)=\frac{C}{\tau *}\left(1+\frac{1}{\beta \tau *}\right) .
$$

Consider the numerical values

$$
C=R 10000, \quad z_{0}=R 2 / h, \quad \mu=0.0002, \quad \sigma=0.1
$$

Then,

$$
\begin{array}{ll}
\mathrm{z}^{*}=4.0415 \mathrm{R} / \mathrm{h}, & \tau^{*}=3053 \mathrm{~h} \\
\mathrm{~K}(\mathrm{z} *)=6.885 \mathrm{R} / \mathrm{h}, & \mathrm{K}\left(\tau^{\star}\right)=7.567 \mathrm{R} / \mathrm{h} .
\end{array}
$$

The expected length of the replacement interval corresponding to $z^{*}$ is

$$
E\left(Y_{Z}\left(Z^{*}\right)\right)=\frac{\ln 2.02075}{0.0002}=3517 \mathrm{~h}
$$

\section{Example 2 Let}

$$
Z(t) \equiv W(t) .
$$

Then,

$$
K(z)=z+\frac{\mu C}{z} \text { and } K(\tau)=\mu \tau+\frac{C}{\tau} \text {. }
$$


Therefore, in view of

$$
\begin{aligned}
& \mathrm{Z} *=\sqrt{\mu \mathrm{C}} \text { and } \tau *=\sqrt{\mathrm{C} / \mu}, \\
& \mathrm{K}(\mathrm{Z} *)=\mathrm{K}(\tau *)=\sqrt{\mu \mathrm{C}} .
\end{aligned}
$$

Moreover,

$$
E\left(Y_{Z}(Z *)\right)=\frac{\sqrt{\mu C}}{\mu}=\sqrt{\frac{C}{\mu}}=\tau *
$$

Corollary If the repair cost rate is given by a Wiener process with positive drift, then, from the cost point of view, policy I and the economic lifetime policy are equivalent replacement strategies. Moreover, the corresponding expected replacement intervals coincide.

\section{Example 3 Let}

$$
Z(t)=z_{0}(W(t))^{2}, \quad t \geq 0 .
$$

Since $Z(t)=z$ holds if and only if $W(t)=\sqrt{z / z_{0}}$, the expected first-passage time is given by

$$
E\left(Y_{Z}(z)\right)=\frac{\sqrt{z / z_{0}}}{\mu}
$$

Therefore,

$$
K(z)=z+\frac{\mu C}{\sqrt{z / z_{0}}} .
$$

The optimal limit $z=z^{*}$ is

$$
z *=\left[\frac{z_{0} \mu^{2} C^{2}}{4}\right]^{1 / 3}
$$

Further,

$$
K(z *)=\frac{3}{2}\left[2 z_{0} \mu^{2} C^{2}\right]^{1 / 3}
$$

Comparision with the economic lifetime approach: Note that the expected total repair cost in $[0, t]$ is given by

$$
E(A(t))=E\left[z_{0} t(W(t))^{2}\right]=z_{0} t\left[\sigma^{2} t+\mu^{2} t^{2}\right] .
$$

This result can be easily obtained by making use of the moment generating function of the normal distribution. Hence, applying the replacement interval $\tau$, the maintenance cost rate is 


$$
K(\tau)=z_{0}\left[\sigma^{2} \tau+\mu^{2} \tau^{2}\right]+\frac{C}{\tau}
$$

The economic lifetime in case of $\sigma^{2}=0$ (deterministic repair cost development) is

$$
\tau *=\left[\frac{\mathrm{C}}{2 z_{0} \mu^{2}}\right]^{1 / 3} \text {. }
$$

Thus,

$$
K(\tau * \mid \sigma=0)=\frac{3}{2}\left[2 z_{0} \mu^{2} C^{2}\right]^{1 / 3}=K(z *)
$$

It follows that

$$
K(\tau * \mid \sigma>0)>K(\tau * \mid \sigma=0)=\frac{3}{2}\left[2 z_{0} \mu^{2} C^{2}\right]^{1 / 3}=K(z *) .
$$

Therefore, in this special case, applying the economic lifetime is not efficient.

\section{LIMIT FOR THE TOTAL REPAIR COST}

Since $Z(t)=A(t) / t$ with $A(t)$ being the total repair costs in $[0, t]$, it seems obvious to schedule replacements on the basis of the development of the stochastic process $\{A(t), t>0\}$. This leads to the following maintenance policy:

Policy II The system is replaced as soon as $\mathrm{A}(\mathrm{t})$ reaches or exceeds a positive level a.

The corresponding maintenance cost rate is

$$
K(a)=\frac{a+c}{E\left(Y_{A}(a)\right)}
$$

where $Y_{A}(a)$ is the first passage time of the stochastic process $\{A(t), t \geq 0\}$ with respect to $a$.

\section{Example 4 Let}

$$
A(t)=a_{0}(W(t))^{2}, \quad a_{0}>0
$$

Then, according to (3),

$$
E\left(Y_{A}(a)\right)=\frac{\sqrt{a / a_{0}}}{\mu}
$$

Hence,

$$
K(a)=\frac{a+C}{\sqrt{a / a_{0}}} \mu .
$$


The optimal limit $a=a^{*}$ satisfies $d K(a) / d a=0$. It follows that

$$
\mathrm{a} *=\mathrm{C} \text {. }
$$

Thus, it is cost-optimal to replace the system as soon as the total repair cost in $[0, t]$ is equal to the replacement cost. The minimal maintenance cost rate for this policy is

$$
\mathrm{K}(\mathrm{a} *)=2 \mu \sqrt{\mathrm{a}_{0} \mathrm{C}} \text {. }
$$

Comparision with the economic lifetime approach: When applying the replacement interval $\tau$, the maintenance cost rate becomes

$$
K(\tau)=a_{0} \sigma^{2}+a_{0} \mu^{2} \tau+\frac{C}{\tau} .
$$

This follows from (see (4))

$$
E\left[(W(t))^{2}\right]=\sigma^{2} t+\mu^{2} t^{2} .
$$

Hence the economic lifetime is

$$
\tau *=\frac{1}{\mu} \sqrt{\frac{\mathrm{C}}{\mathrm{a}_{0}}} .
$$

The corresponding minimal maintenance cost rate is

$$
K(\tau *)=a_{0} \sigma^{2}+2 \mu \sqrt{a_{0} C} .
$$

Obviously, for all parameter combinations with $\sigma^{2}>0$,

$$
\mathrm{K}(\mathrm{a} *)<\mathrm{K}(\tau *) \text {. }
$$

\section{REFERENCES}

[1] F. BEICHELT, A unifying treatment of replacement policies with minimal repair, Naval Research Logistics 40, 51-67 (1993).

[2] F. BEICHELT and L. P. FATTI, Stochastic Processes for Engineers, Gordon and Breach, New York, Berlin (1999).

[3] S. CHHIKARA and J. L. FOLKS, The Inverse Gaussian Distribution, Marcel Dekker, Inc., NewYork, Basel (1988).

[4] J. C. R. CLAPHAM, Economic life of equipement, Oper. Res. Quart. 8, 181-190 (1957).

[5] R. W. DRINKWATER and N. V. HASTINGS, An economic replacement model, Oper. Res. Quart.,18, 59-71 (1967). 
[6] P. GARDENT and L. NONANT, Entretien et renouvellement d'un parc de machines, Revue Franch. Rech. Operat.,7, 5-19 (1963).

[7] V. L. SMITH, Economic equipment policies: An evaluation, Management Science, 4, 20-27 (1957).

[8] D. J. WHITE, Repair limit replacement, OR Spektrum, 11, 143-149 (1989).

\section{Acknowledgement}

The author thanks the editor and an anonymous referee for many hints which substantially improved the quality of the paper. 